\title{
Science with Micro-X: the TES Microcalorimeter X-ray Imaging Rocket
}

E. Figueroa-Feliciano, S. R. Bandler, M. Bautz, K. R. Boyce, G. V. Brown, S. Deiker, W. B. Doriese, K. Flanagan, M. Galeazzi, G. C. Hilton, U. Hwang, K. D. Irwin, T. Kallman, R. L. Kelley, C. A. Kilbourne, S. Kissel, A. Levine, M. Loewenstein, D. Martinez-Galarce, D. McCammon, R. Mushotzky, R. Petre, F. S. Porter, C. D. Reistema, T. Saab, P. Serlemitsos, N. Schulz, R. Smith, J. N. Ullom

May 25, 2006

SPIE Astronomical Telescopes and Instrumentation Orlando, FL, United States May 24, 2006 through May 31, 2006 
This document was prepared as an account of work sponsored by an agency of the United States Government. Neither the United States Government nor the University of California nor any of their employees, makes any warranty, express or implied, or assumes any legal liability or responsibility for the accuracy, completeness, or usefulness of any information, apparatus, product, or process disclosed, or represents that its use would not infringe privately owned rights. Reference herein to any specific commercial product, process, or service by trade name, trademark, manufacturer, or otherwise, does not necessarily constitute or imply its endorsement, recommendation, or favoring by the United States Government or the University of California. The views and opinions of authors expressed herein do not necessarily state or reflect those of the United States Government or the University of California, and shall not be used for advertising or product endorsement purposes. 


\title{
Science with Micro-X: the TES Microcalorimeter X-ray Imaging Rocket
}

E. Figueroa-Feliciano ${ }^{a, b}$, S. R. Bandler ${ }^{c, d}$, M. Bautz ${ }^{b}$, K. Boyce $^{c}$, G. Brown $^{e}$, S. Deiker ${ }^{f}$,

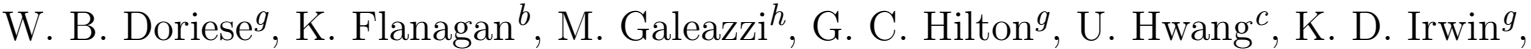
T. Kallman ${ }^{c}$, R. L. Kelley ${ }^{c}$, C. A. Kilbourne ${ }^{c}$, S. Kissel $^{b}$, A. Levine ${ }^{b}$, M. Loewenstein $^{c, d}$, D. Martinez-Galarce $f$, D. McCammon ${ }^{i}$, R. Mushotzky ${ }^{c}$, R. Petre ${ }^{c}$, F. S. Porter ${ }^{c}$,

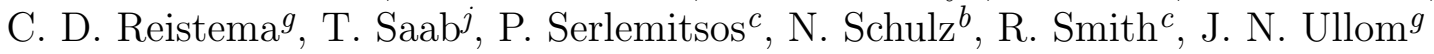

${ }^{a}$ Department of Physics, Massachusetts Institute of Technology, Cambridge, MA 02139, USA;

${ }^{b}$ MIT Kavli Institute for Astrophysics and Space Research, Cambridge, MA 02139, USA;

${ }^{c}$ NASA Goddard Space Flight Center, Greenbelt, MD 20770, USA;

${ }^{d}$ University of Maryland, Department of Astronomy, College Park, MD 20742, USA;

${ }^{e}$ High Energy Density Physics and Astrophysics Division, Lawrence Livermore National Laboratory, Livermore, CA 9455;

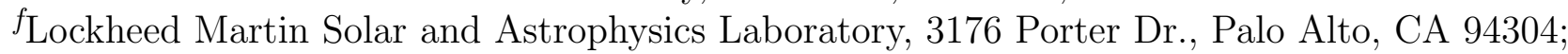

${ }^{g}$ National Institute of Standards and Technology, 325 Broadway, Boulder, CO 80305, USA;

${ }^{h}$ University of Miami, Department of Physics, Coral Gables, FL 33146, USA;

${ }^{i}$ Department of Physics, University of Wisconsin-Madison, Madison, WI 53706, USA;

${ }^{j}$ Deptartment of Physics, University of Florida, Gainsville, FL 32611, USA

\begin{abstract}
Micro-X is a proposed sounding rocket experiment that will combine a transition-edge-sensor X-ray-microcalorimeter array with a conical imaging mirror to obtain high-spectral-resolution images of extended and point X-ray sources. We describe the payload and the science targeted by this mission including the discussion of three possible Micro$\mathrm{X}$ targets: the Puppis A supernova remnant, the Virgo Cluster, and Circinus X-1. For example, a Micro-X observation of the bright eastern knot of Puppis A will obtain a line-dominated spectrum with 90,000 counts collected in 300 seconds at $2 \mathrm{eV}$ resolution across the $0.3-2.5 \mathrm{keV}$ band. Micro-X will utilize plama diagnostics to determine the thermodynamic and ionization state of the plasma, to search for line shifts and broadening associated with dynamical processes, and seek evidence of ejecta enhancement. For clusters of galaxies, Micro-X can uniquely study turbulence and the temperature distribution function. For binaries, Micro-X's high resolution spectra will separate the different processes contributing to the Fe $\mathrm{K}$ lines at $6 \mathrm{keV}$ and give a clear view of the geometry of the gas flows and circumstellar gas.
\end{abstract}

Keywords: Supernova Remnants, Clusters of Galaxies, X-ray binaries, X-rays, Microcalorimeters, Sounding Rockets

\section{INTRODUCTION}

The promise of imaging, high resolution X-ray spectroscopy remains unfulfilled. The unfortunate loss of the Suzaku X-ray Spectrometer (XRS) and the delay of Constellation-X leaves the X-ray astronomy community without a certain prospect of fulfilling the original AXAF scientific objectives for many years. The Japanese New X-ray Telescope (NeXT) mission is the most likely next advance, but its launch date is still uncertain. The lack of flight opportunities is especially frustrating given the technical readiness of X-ray microcalorimeters. We propose to fill this gap in scientific capability with Micro-X, a sounding rocket payload that combines an X-ray microcalorimeter with an imaging mirror to offer breakthrough science from high spectral resolution observations of extended X-ray sources.

E. Figueroa-Feliciano's e-mail: enectali@mit.edu 


\begin{tabular}{|l|rl|}
\hline Energy Resolution $\Delta E$ & 2 & $\mathrm{eV} \mathrm{FWHM}$ \\
\hline Effective Area @ 1 keV & 200 & $\mathrm{~cm}^{2}$ \\
\hline Point Spread Function & 2.5 & $\mathrm{arcmin}$ \\
\hline Mirror Focal Length & 2.1 & $\mathrm{~m}$ \\
\hline FOV & 14.4 & $\mathrm{arcmin}$ \\
\hline Size of Detector Array & $11 \times 11$ & \\
\hline Pixel Size & 800 & $\mu \mathrm{m}$ \\
& 1.3 & $\mathrm{arcmin}$ \\
\hline Bandpass & $0.3-2.5$ & $\mathrm{keV}$ \\
\hline
\end{tabular}

Table 1. Micro-X Technical Specifications

Micro-X will consist of a Transition Edge Sensor (TES) microcalorimeter array at the focus of a flight-proven conical imaging mirror. Progress in the last decade now allows routine fabrication of microcalorimeter arrays with energy resolutions from 2.5-4.7 eV FWHM reported by various groups; ${ }^{1,2}$ these approach the theoretical resolutions of $1-2 \mathrm{eV} .^{3}$

Micro-X will offer a unique combination of bandpass, collecting area, and spectral and angular resolution. The spectral resolution across the $0.3-2.0 \mathrm{keV}$ band will be $2 \mathrm{eV}$. The angular resolution across the 14.4 arc minute field of view will be 2.5 arc minutes. The effective area, $200 \mathrm{~cm}^{2}$ at $1 \mathrm{keV}$, is sufficient to provide observations of unprecedented quality of numerous cosmic X-ray sources, discrete and extended, even in a brief sounding rocket exposure.

Our scientific program will initially focus on extended sources, for which high-spectral-resolution, wide-energybandwith observations were hitherto impossible. For supernova remnants the Micro-X objectives are to utilize numerous plasma diagnostics to determine the thermodynamic and ionization state of the plasma, to search for line shifts and broadening associated with dynamical processes, and seek evidence of ejecta enhancement. For clusters of galaxies, Micro-X can uniquely study turbulence and the temperature distribution function. For our initial flight, we will observe the bright eastern knot in the Puppis A remnant, a site of complex cloud-shock interactions and ejecta enrichment. Other SNRs for which breakthrough observations are possible include the Cygnus Loop and Cas A. The center of the Virgo Cluster is the highest priority cluster target. Eventually the payload can be reconfigured with the ASCA flight spare mirror to measure the velocity of accreting and outflowing gas in X-ray binary systems.

\section{THE MICRO-X PAYLOAD}

We believe the current generation of TESs are at a technology readiness level ideally suited for a rocket flight. Micro-X is the next logical step to synthesize TES technologies around a flight system. In this section we give a brief overview of the payload. Table 1 summarizes the technical specifications of Micro-X.

\subsection{Detectors for Micro-X}

Our reference design for Micro-X consists of an $11 \times 11$ array of $0.7 \mathrm{~mm} \times 0.7 \mathrm{~mm}$ pixels on an $0.8 \mathrm{~mm}$ pitch. This odd size array scale was chosen as a reference for modeling based on the availability of a $4 \times 32$ SQUID MUX system. The physical array may be $12 \times 12$, or even $16 \times 16$. Coupled to the $2.1 \mathrm{~m}$ focal length mirror, each array pixel will be 1.31 arcmin on the sky, a good match to the 2.5 arcmin imaging of the mirror.

We have chosen to limit the complexity of the arrays by foregoing the use of the high-fill-factor X-ray absorbers under development for Constellation-X. For the soft X-ray array, the TES sensors themselves have sufficient quantum efficiency, and for the broad band array we will apply a bismuth absorber of the same area as the TES directly to the sensor. This creates a "waffle-like" focal plane with dead region at the wiring gap between pixels (see Figure 1). The $77 \%$ fill factor is adequate for our observations. We will prevent X-ray interaction in the electrical leads and silicon structure through use of a mask. 


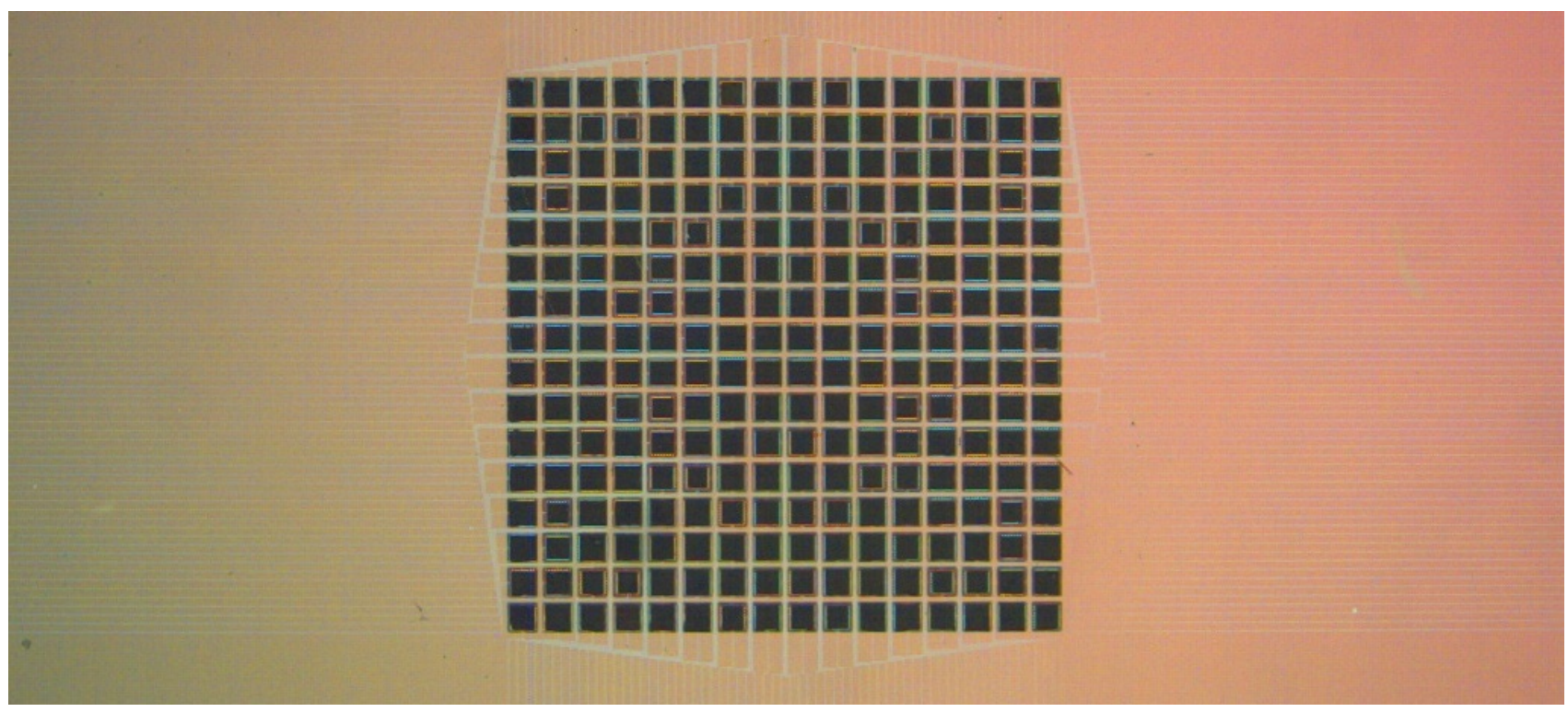

Figure 1. A NIST TES $16 \times 16$ array. The dark squares are the TESs. These arrays are designed for Constellation-X and are built at a $350 \mu \mathrm{m}$ pitch (with $250 \mu \mathrm{m}$ TESs). The Micro-X array will use the same "waffle" design.

We can apply simple scaling arguments to predict the performance of these arrays. Given that the heat capacity is largely determined by the TES area, the main variable to adjust is the sensitivity, $\alpha{ }^{1}$ demonstrated a $0.4 \mathrm{~mm}$ device made at NIST with $3-\mathrm{eV}$ resolution across a broad band. If we were to increase the heat capacity by 3 (through increasing the area of the TES) and increase $\alpha$ by 3, we would obtain a 3.4-eV device with a saturation energy of $10 \mathrm{keV}$ (Resolution scales as $\sqrt{C / \alpha}$ for an ideal device, but the degradation predicted accounts for an excess noise term that depends on $\alpha^{4}$ ). Increasing $\alpha$ by a factor of 6 would produce a device with $1.9-\mathrm{eV}$ resolution and a saturation energy of $5 \mathrm{keV}$. The value of $\alpha$ is tuned through adjusting the density of perpendicular normal metal strips applied to the surface of the TES. ${ }^{4}$

The detectors will be housed in an enclosure with an X-ray aperture. This detector assembly provides mechanical and thermal staging of the TES array and the front-end SQUID amplifiers. It includes suspension systems, wiring interfaces, and magnetic shielding.

\subsection{Readout Electronics}

We will read out the detector array using a NIST time-division SQUID multiplexer. ${ }^{5-7}$ In a time-division multiplexer, amplifiers are turned on one at a time, with switching occurring fast enough that each detector is sampled faster than the detector time constants of interest. The signals from all first-stage amplifiers (only one of which is on at a time) are summed. The NIST scheme takes this idea to two dimensions, so that in an array, rows of SQUID amplifiers are turned on sequentially, and each column has its own series-array amplifier. In this case, the number of wires to the cold stage necessary to read out an $N \times N$ detector array scales roughly as $2 N$, rather than $N^{2}$ for the brute-force readout technique. NIST recently demonstrated that the SQUID TDM can read out the required number of detectors with sufficient energy resolution for Micro-X. ${ }^{8}$

\subsection{Cryogenic Systems}

The Micro-X ADR cryogenic system will be based on the very successful X-ray Quantum Calorimeter (XQC) sounding rocket refrigerator. ${ }^{9,10}$ The Micro-X cryostat will be substantially similar to the XQC system. Some modifications are required by the TES detector system that complicate the cryostat design, but at the same time the more restricted field of view of the Micro-X instrument make some parts of the design simpler. The biggest challenge in the Micro-X cryogenic design is to provide near-zero magnetic field regions in the instrument around the TES detectors and the SQUID amplifiers. We will design an optimal, staged magnetic isolation system for 


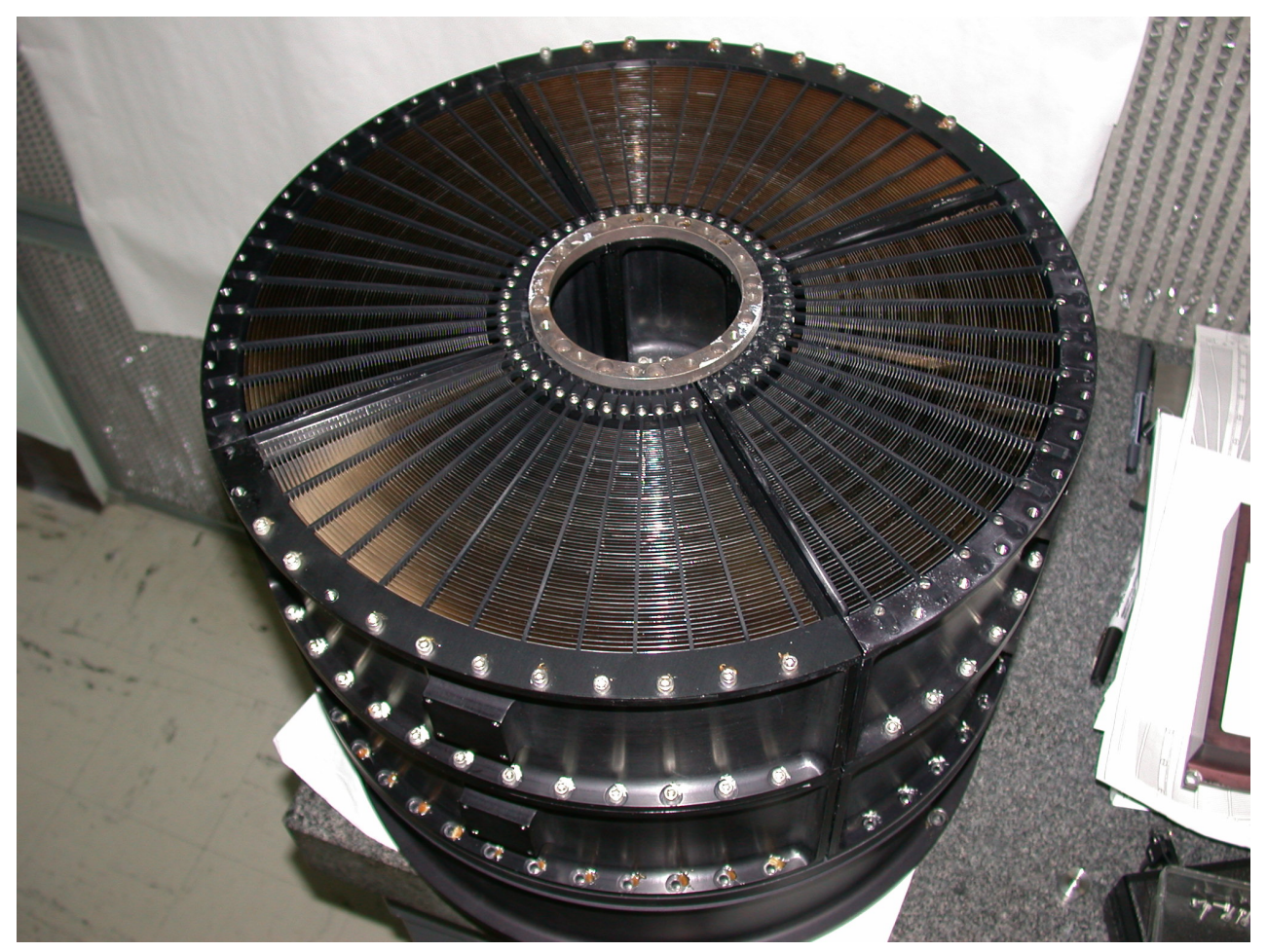

Figure 2. The Micro- $\mathrm{X}$ mirror.

the detectors and amplifiers. Local superconducting shields will be designed for the detector system and SQUIDS that minimize the susceptibility to trap flux.

\subsection{Mirror and optical bench}

The Micro-X instrument will use the imaging mirror from the Supernova X-Ray Spectrometer (SXS), a sounding rocket instrument flown by the GSFC group in 1988 to observe SN 1987A (Figure 2). The mirror is the first segmented, thin foil conical mirror ever to have flown. The foil reflectors were lacquer coated using the approach used for the BBXRT and ASCA mirrors. It has a $2.1 \mathrm{~m}$ focal length and a $40 \mathrm{~cm}$ diameter with 70 nested shells configured in quadrants. The high filling factor of the thin-foil implementation provides $>300 \mathrm{~cm}^{2}$ of area at $1 \mathrm{keV}$ and a broad energy response. After including filter transmission and detector fill fraction we still have $>200 \mathrm{~cm}^{2}$ of effective area (Figure 3). This effective area was used for the simulations in this paper. The optic's measured angular resolution is $\sim 2.5^{\prime}$ (half power diameter).

If a bandpass extension beyond $6 \mathrm{keV}$ is desired for study of X-ray binaries, the ASCA flight spare mirror can be installed. Use of this mirror would require lengthening of the optical bench to accommodate the longer focal length $(3.5 \mathrm{~m})$. The ASCA mirror would provide Micro-X with $\sim 100 \mathrm{~cm}^{2}$ at $6 \mathrm{keV}$.

\section{SCIENCE WITH MICRO-X}

Much of the detailed physics of astronomical sources is accessible only through spectroscopy. In particular a wide variety of kinematic, temperature, ionization, density and abundance diagnostics are available only at moderate to high spectral resolution $(E / d E=R>20)$. Such data have long been available at optical and radio wavelengths, but only the latest generation of missions have made them available to X-ray astronomers. Besides $\mathrm{H}$ and $\mathrm{He}$, all of the abundant elements produce X-ray emission - -in particular, the K-shell transitions of $\mathrm{C}$ through Fe, as well as the L-shell transitions of Si through Fe, and the M shell transitions of Fe and Ni. Cosmic X-ray spectra can therefore be quite rich, with a multitude of spectral lines conveying fundamental physical information. In contrast to spectra at other wavebands, a single X-ray spectrum can include all the extremes 


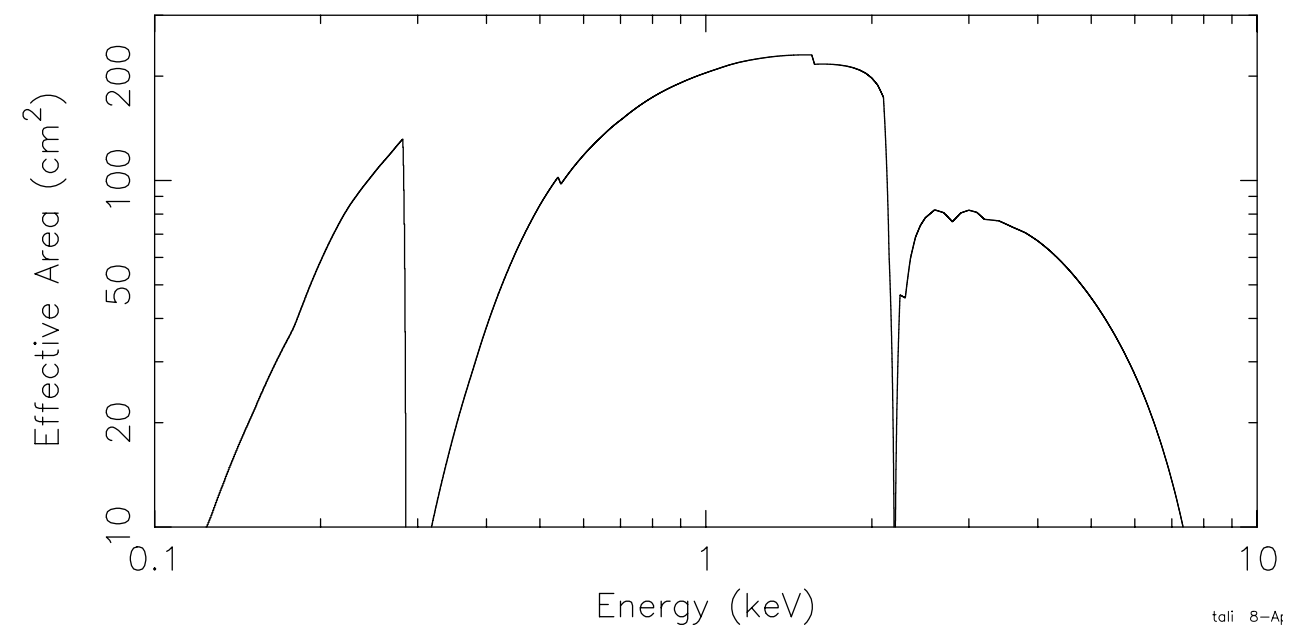

Figure 3. The Micro-X effective area, including the mirror, the blocking filters, and the TES quantum efficiency and packing fraction.

in the possible charge states of a given element - from fluorescent lines in cold neutral material to lines of the hydrogren-like ion of Fe.

For high spectral resolution data, the technique of plasma diagnostics provides a means for unraveling the plasma conditions in a relatively model-free manner. The key requirement is the ability to measure fluxes of individual X-ray lines in order to place physical constraints on the emitting plasma. By using ratios of line fluxes, uncertainties in the distance to the source are eliminated. By taking pairs of lines of similar energy from the same ion, uncertainties in column density are minimized, and plasma temperatures may be constrained. Lines from different ionization states provide information on ionization balance, and lines from different elements provide abundance information.

For point sources, the Chandra and XMM-Newton grating spectrometers have amply demonstrated the power of $R>20$ spectroscopy. ${ }^{11}$ The results include measuring precise chemical abundances in stellar coronae, ${ }^{12}$ solidifying the cooling flow mystery in clusters of galaxies ${ }^{13}$ and discovering massive outflowing winds in active galaxies. ${ }^{14}$ While moderate resolution X-ray CCD spectra have long been available for extended sources, high resolution spectra are currently available only in a limited way with Chandra and XMM-Newton. Because gratings are dispersive spectrometers, they cannot achieve the spectral resolution needed to measure individual line fluxes if the source has a significant angular extent. At best, the XMM RGS spectra are an improvement over CCD spectrometers only for sources smaller than $3^{\prime}$ in size. Many of the brightest extended sources in the sky are much larger than this, and are thus inaccessible for study at high spectral resolution. This gap in our capability should have been filled by the Suzaku XRS, but its unfortunate demise has prevented this advancement from coming to fruition.

The vast scientific potential for spectroscopy is discussed in detail by Paerels. ${ }^{11}$ We highlight a few examples. (1) Resolve the He-like triplet lines of the abundant elements: These lines are strongly sensitive to electron density, temperature, and ionization conditions in the emitting plasma. (2) Sensitivity to velocity structure: For example, $500 \mathrm{~km} / \mathrm{s}$ turbulent velocities are predicted in clusters of galaxies and will be imprinted in line profiles and energy shifts. ${ }^{15}$ (3) Distinguish the source of ionization of the plasma: The exact line energies and strengths will distinguish between scenarios such as photoionization, collisional and shock ionization. (4) (Partially) resolve the vast sea of $\mathrm{Fe} \mathrm{L}$ shell lines in the $0.7-1.4 \mathrm{keV}$ band: These lines dominate the spectrum of collisionally ionized plasmas with $0.4<k T<2 \mathrm{keV}$, and are strong even when lower-Z elements are fully stripped. For collisionally ionized plasmas, this complex samples a wide range in temperature and has significant diagnostic sensitivity (see Figure 6).

The Micro-X mission as proposed is designed to obtain high resolution data that will help to answer such questions for the brightest large extended objects in the sky, and restore some of the science lost to Suzaku. Here 


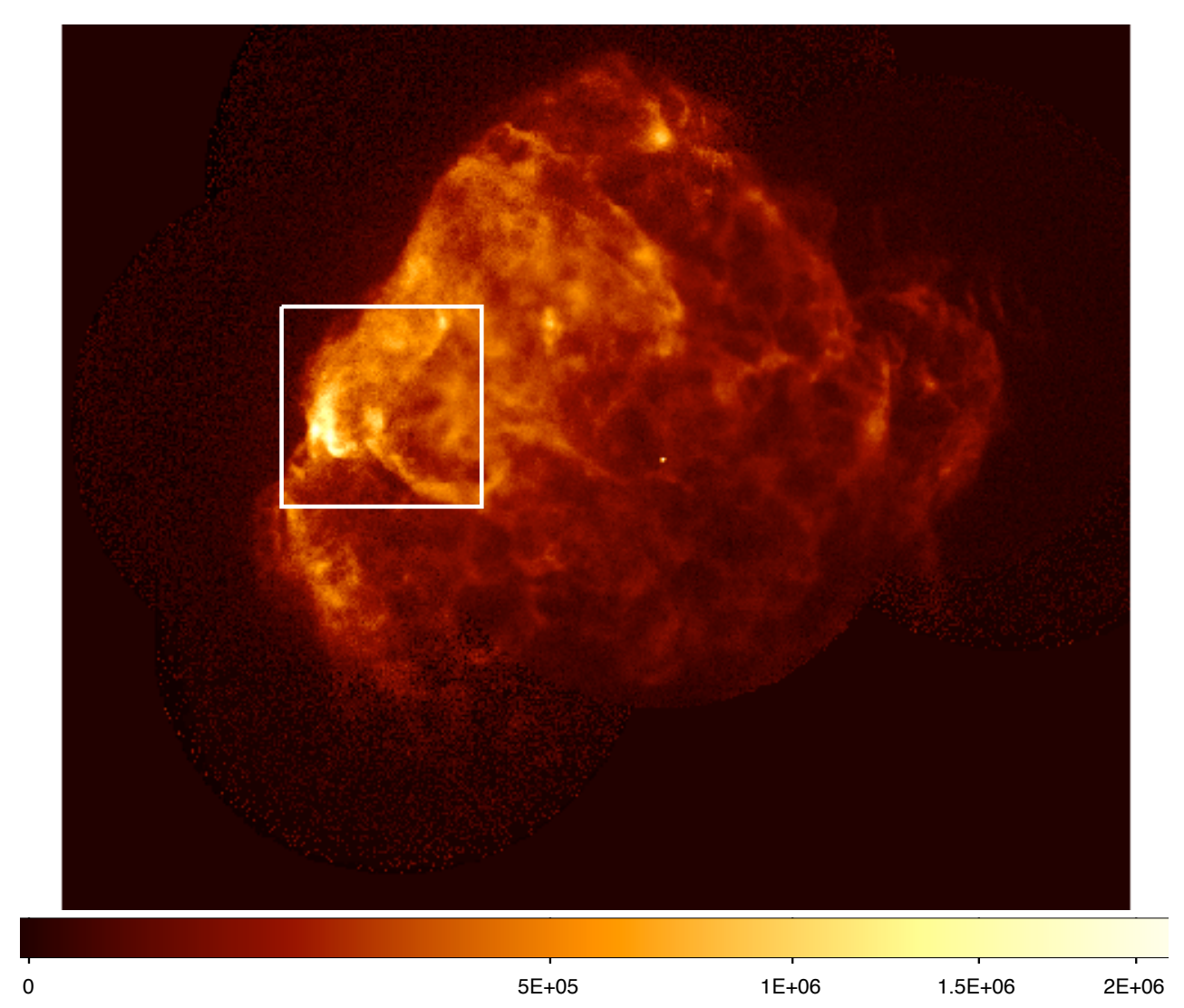

Figure 4. The Puppis A supernova remnant. The box represents the $14.4^{\prime}$ FOV for observation of the Bright Eastern Knot by Micro-X.

we give two detailed examples of this science: the Puppis A supernova remnant and the giant elliptical galaxy M87 at the core of the Virgo cluster. With these data, we ascertain the temperature and ionization state of the $\mathrm{X}$-ray emitting gas in Puppis A. This is crucial to unravelling the presence of supernova ejecta in this remnant, the turbulent processes that take place as a molecular cloud is torn apart by shocks, and shock-induced star formation. In the case of M87, we can search for the kinematic signature of the heating of the X-ray emitting gas that must be taking place, ${ }^{16}$ determine precise chemical abundances for oxygen, nitrogen and neon, and search for the origin of the lack of cooling in the gas below $0.6 \mathrm{keV}$. Similar scientific studies would be possible for $\sim 2-3$ other clusters of galaxies and several other supernova remnants, such as Cas A, Tycho's SNR and the Cygnus Loop.

With an upgrade of the mirror to achieve $100 \mathrm{~cm}^{2}$ of effective area at $6 \mathrm{keV}$, high resolution studies of $6 \mathrm{keV}$ emission from accretion disks around compact objects can be undertaken.

\subsection{Galactic Supernova Remnants}

The highest priority candidate for the initial Micro-X observation is the Bright Eastern Knot region of the Puppis A supernova remnant (Figure 4). Puppis A is a bright Galactic supernova remnant that was the target of one of the earliest successful high-resolution spectral observations with the Einstein Focal Plane Crystal Spectrometer. ${ }^{17,18}$ These observations were of limited energy range and sensitivity, however, and the spectral resolution of this dispersive instrument was compromised by the large angular scale of the X-ray emission.

As a middle-aged remnant, Puppis A straddles the division between young objects that are totally dominated by their supernova debris, or ejecta, and older remnants that have lost all trace of their ejecta. Despite a rather advanced estimated age of 4000 years, it contains both optical and X-ray evidence for supernova ejecta. However, 


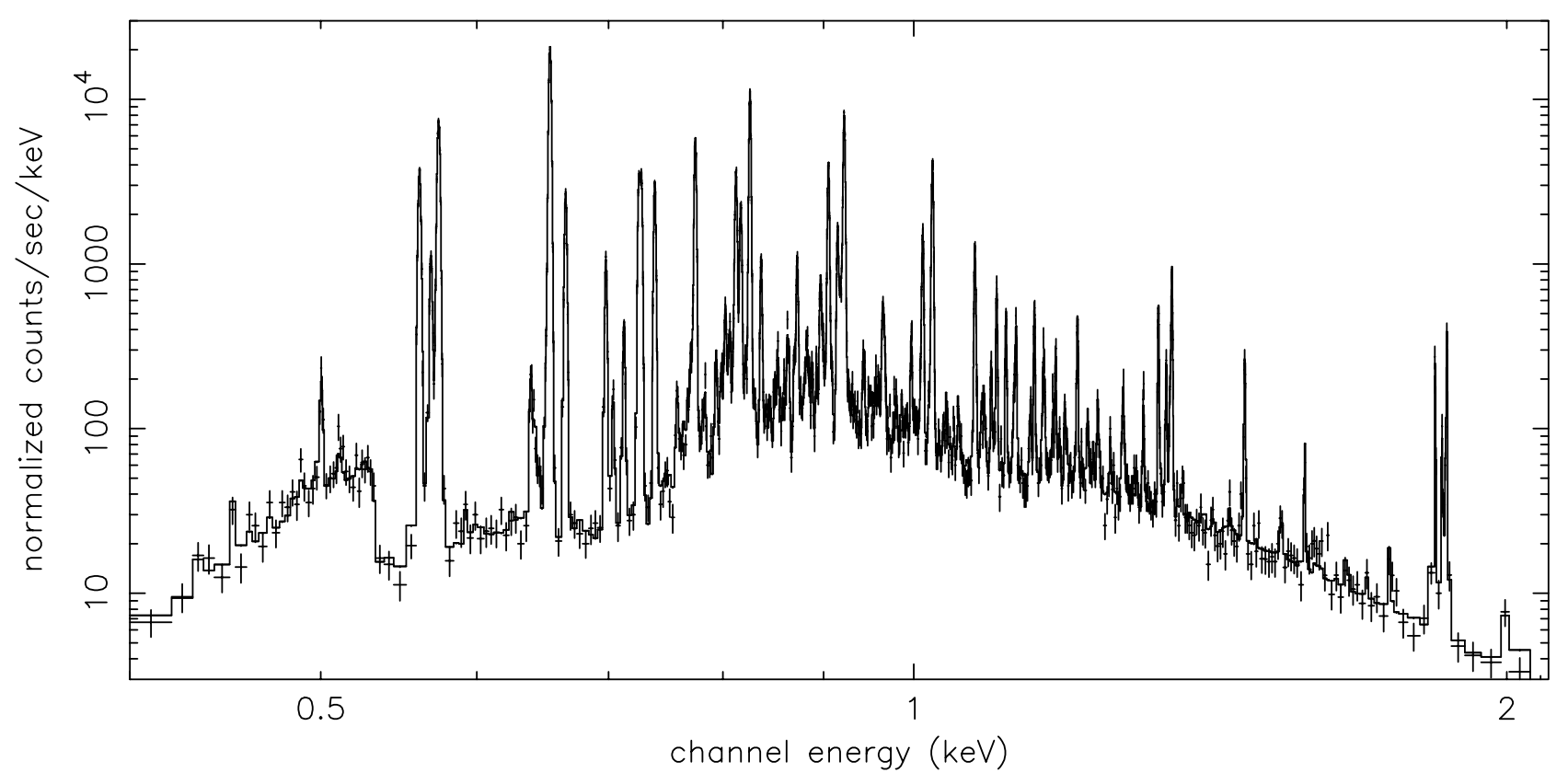

Figure 5. Simulation of the integrated Puppis A spectrum assuming 300 sec observation by Micro-X with 2 eV resolution. There are 92,900 counts in this simulation. The effective area curve used for this simulation, which includes mirror, filters, and detector efficiencies is shown in Figure 3.

it is also very strongly shaped by its interactions with a complex interstellar environment. The Bright Eastern Knot (BEK) region of Puppis A is the brightest, and perhaps most complex example of these interactions, with multiple shocked clouds plus evidence for ejecta enrichment in its vicinity.

The main science goals of the Micro-X observation are first to unravel the kinematic, temperature, and ionization characteristics of the cloud-shock interactions at the BEK, and second to address the issue of ejecta enrichment of the X-ray emitting gas. Crucial to both of these efforts will be ascertaining the temperature distribution of the X-ray emitting gas. This is important for understanding ejecta enrichment because multitemperature gas can mimic enriched abundances in lower resolution spectra if too simple a temperature structure is assumed. A simulated $300 \mathrm{~s}$ observation with a 14.4 arcmin field of view at the BEK is shown in Figure 5. The model in Figure 5 is a three-temperature nonequilibrium ionization thermal model that approximates the integrated spectrum of a smaller field centered at the BEK observed with Chandra, and is scaled to the Micro-X field of view using the X-ray images obtained with the ROSAT X-ray Observatory.

\subsubsection{Plasma Conditions and Shocked Clouds}

The high quality of the Micro-X spectrum will provide a detailed look at the plasma conditions in Puppis A. Chandra observations indicate a rather complex temperature structure in the BEK region. ${ }^{19}$ With $2 \mathrm{eV}$ energy resolution, we will be able to diagnose the temperature structure in the X-ray emitting gas by identifying the Fe L lines that are present in the spectrum. Figure 6 shows a detail view of the Fe L region near $0.55-0.95 \mathrm{keV}$ in the simulated spectrum, where lines of Fe XVII to Fe XIX are readily identified. The Fe L lines are powerful temperature diagnostics, as the relative emissivity curves of various Fe ions are different at different temperatures. The identification of the Fe ions and a measurement of their relative flux in the Micro-X spectrum will thus place direct constraints on the temperatures present in the plasma.

Supernova remnant plasmas are typically not in ionization equilibrium for their temperatures, because of their low densities. The supernova shocks (the two main ones being the forward shock associated with ambient material and the reverse shock associated with ejecta) heat the gas quickly, but the gas ionizes slowly by electronion collisions. The ionization structure is generally time-dependent throughout the lifetime of the X-ray emitting remnant. Diagnostics of nonequilibrium ionization are provided by the line intensity ratios of the resonance, 


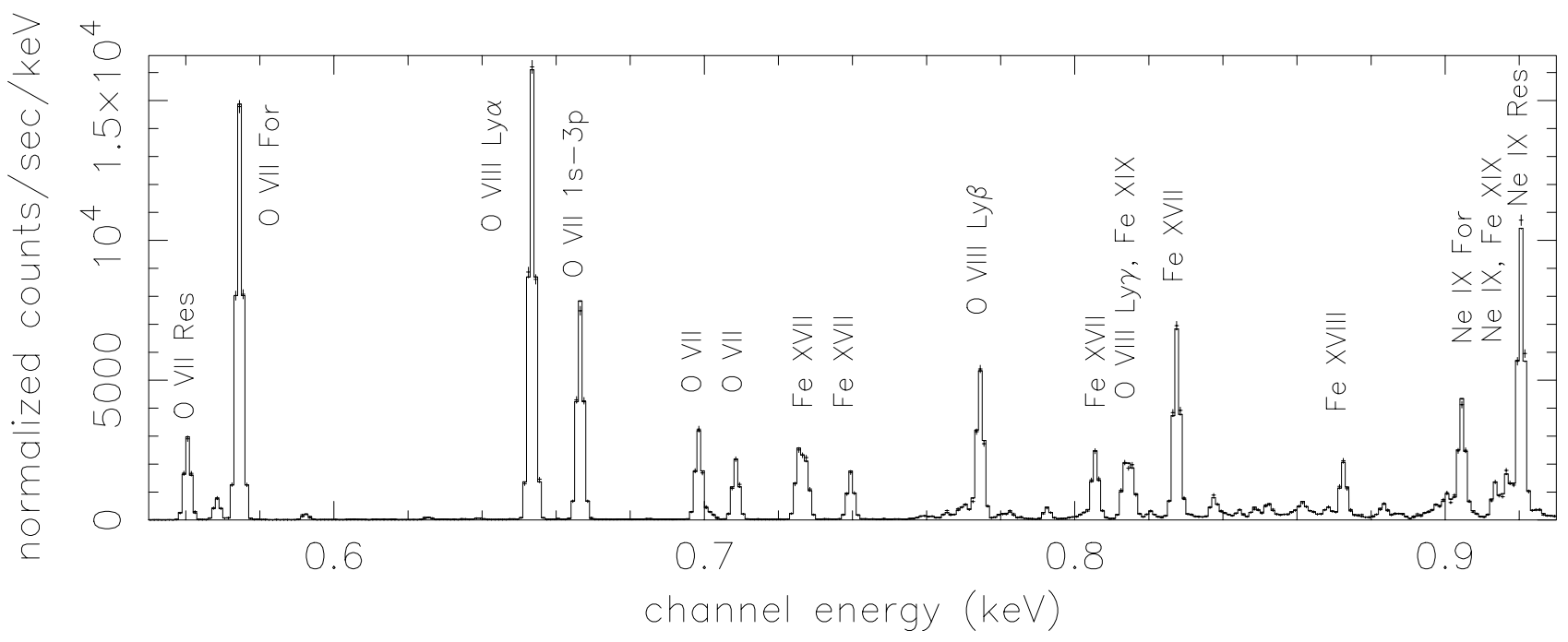

Figure 6. Detail of Puppis A simulated spectrum from Figure 5.

forbidden, and intercombination triplet in He-like ions, as well as low ion species that may contribute a lowenergy component to the main He-like blends and Fe XVI inner-shell transitions that may be visible near Fe XVII lines.

In addition to temperature and ionization information, another important diagnostic of the cloud-shock interactions will be given by the line profiles. A cloud that has encountered a shock will subsequently be torn apart by hydrodynamic instabilities. ${ }^{20}$ Chandra identified the inner region of the BEK to be a cloud that is in an advanced phase of this very process; ${ }^{19}$ indeed it is the only identified astrophysical example of such a mature cloud-shock interaction to date. Line energy shifts and line widths will be imprinted by these turbulent processes. The Micro-X energy resolution and high signal to noise data will allow measurement of turbulent velocities down to $\pm 10 \mathrm{~km} / \mathrm{s}$, which is the statistical resolution of our data, though systematic effects (due to calibration and gain uncertainties) may be a factor of 2 larger.

Because the BEK is a clump (or clumps) of interstellar material being overtaken by the shock, it is a promising region to find neutral material, and thus to observe the spectral signatures of charge exchange reactions. In its simplest form, astrophysical charge exchange (CEX) is the radiationless transfer of an electron from a neutral atom (usually $\mathrm{H}$ or $\mathrm{He}$ ) to a highly charged ion, with subsequent emission of an X-ray photon as the transferred electron decays to the ground state. In the case of CEX between bare ions and neutral atoms, the high-n transitions are considerably stronger than in the case of collisional excitation, ${ }^{21}$ resulting in anomalous Lyman series line intensity ratios. Such distinct spectral signatures make it possible to identify CEX and distinguish it from emission from a collisional equilibrium plasma, or one dominated by radiative recombination. The strength of the spectral signature can then be used to quantify the amount of neutral gas present, and the relative line intensities (in the case of CEX involving bare ions) used to determine the relative collision speed between the ions and neutrals.

Despite its large cross-section, the normally low abundance of neutral material generally prevents CEX from having a significant effect on the X-ray spectrum for supernova shocks in the sense of modifying the cooling power or total X-ray flux. ${ }^{22}$ CEX would be enhanced, however, by turbulent mixing such as would occur when the shock interacts with a dense interstellar cloud as at the BEK. The general problem of how mixing occurs in a shock-cloud interaction is vital to understanding shock-induced star formation but is also difficult to model. Measurements (or limits) on the amount of CEX emission present in an actual shocked cloud would test such models in a new regime. ${ }^{23}$ 


\subsubsection{Oxygen Enhancement}

An enduring question surrounding Puppis A is the identification of X-ray emitting ejecta. Although the X-ray brightest features in the Puppis-A BEK have been identified as shocked interstellar clouds, ${ }^{24}$ line strengths measured at high spectral resolution by the Einstein FPCS $^{17,18}$ indicated that, despite significant dilution by shocked ambient material, there is evidently an overall oxygen abundance enhancement arising from the presence of shocked oxygen ejecta synthesized by the remnant's massive progenitor.

Subsequent observations with CCD instruments having only moderate spectral resolution have not been able to definitively identify the oxygen ejecta, although observations with ASCA did indicate the presence of $\mathrm{Ne}$ ejecta in the western and southern regions of the remnant. ${ }^{25}$ More recently, moderate spectral resolution observations with the Chandra ACIS instrument of the Bright Eastern Knot in Puppis A suggest that there is oxygen enhancement in localized regions near the shocked interstellar clouds. ${ }^{19}$ It is not surprising that the ejecta should become highly fragmented and localized as the shock is interacting with a highly inhomogeneous environment. Tantalizingly, the optical ejecta knots are mostly contained within an angular distance of some ten arcminutes of the BEK. Line strengths measured directly relative to continuum in this region will unambiguously reveal the presence of ejecta, provided that the field of view captures an area enriched with ejecta and provided that we can be sure of the underlying temperature structure of the gas. The existing CCD spectra do not unambiguously indicate the temperature structure of the gas. We will tackle this problem by combining the analysis of the Micro-X data (which will have $~ 2.5$ arcmin imaging) with ongoing and detailed analysis of data with better angular resolution (but poorer spectral resolution) from XMM-Newton, Chandra, and Suzaku. The high spectral resolution of the Micro-X spectrum will provide the crucial test of the underlying temperature structure of the gas and the measurement of line fluxes relative to the continuum to address the question of the presence of ejecta in Puppis A.

\subsubsection{Other SNR Targets}

There are several other SNR targets for future flights. Simulations of the Cygnus Loop indicate a total of around 6000 counts, most of which are in lines, and allow most of the science described above to be done. Cas A will have 20,000 counts, and other regions of Puppis, as well as Vela, would also be good targets.

\subsection{Clusters of Galaxies}

High resolution X-ray spectroscopy is crucial for understanding the interplay between the complex physical processes occurring at the cores of clusters of galaxies. These include rapid radiative cooling, star formation, AGN-induced feedback, and turbulence. Since this same ensemble of phenomena governs the formation of galaxies and of stars in galaxies, cluster cores serve as astrophysical laboratories with broad fundamental application.

By virtue of its temperature, proximity, and flux, the hot gas in the center of the Virgo Cluster (i.e., that associated with the central galaxy M87) is the ideal cluster target for a mission such as Micro-X with a limited exposure time, modest spatial resolution, good sensitivity, and superb energy resolution at soft X-ray wavelengths. Other targets with similar flux include Ser159 and NGC5044.

Although Micro-X's field of view of 14.4 arcmin will image a large part of M87, we use just a 2 arcmin aperture for our simulations. This is the region dominated by the M87 cooling flow, and we simulate spectra based on the XMM-Newton RGS data analysis of, ${ }^{26}$ and the EPIC analysis of, ${ }^{27},{ }^{28,29}$ and. ${ }^{30}$ These analyses indicated the presence of multiple temperatures over a limited range, and average abundances in roughly solar proportions in this region.

Our goals for a Micro-X observation of Virgo/M87 are to place detailed constraints on the temperature distribution function, reduce the systematic uncertainties in the abundances, measure the degree of turbulence in the plasma, and investigate whether different temperature phases have distinct abundances. The last would provide insight into the interaction and relationship between the different phases, as well as their origin.

The simulated spectra have about 3000 counts in the central $2^{\prime}$ (the total counts for the $14.4^{\prime}$ FOV are about 15000). The turbulent velocity is measured to $\pm 100 \mathrm{~km} / \mathrm{sec}$, and an independent constraint is obtained by examining the level of suppression of resonance scattering of the highest optical depth emission lines. In 


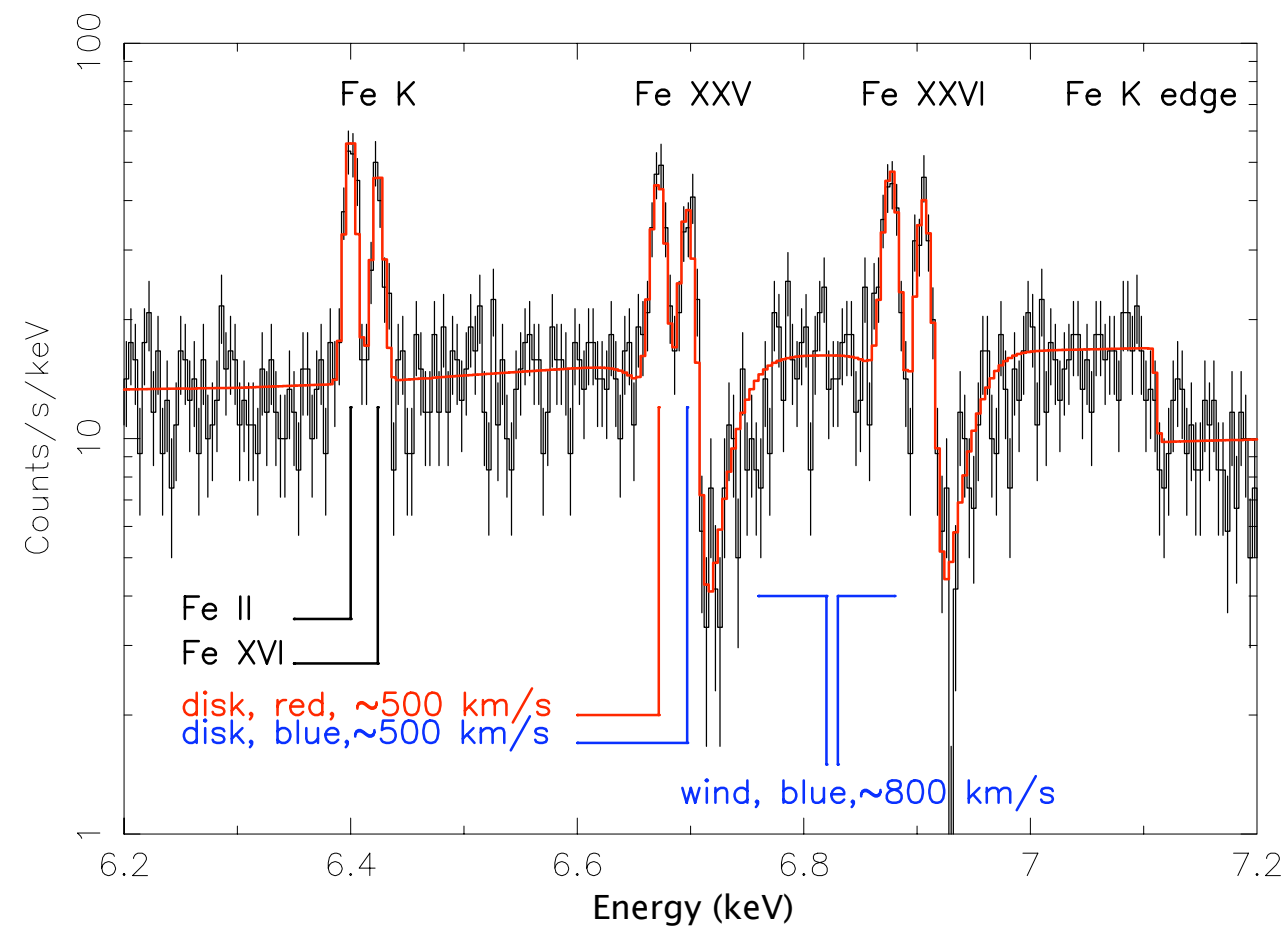

Figure 7. Simulation of the Fe K line region of the ultraluminous X-ray binary Cir X-1 near an X-ray flux of 600 mCrab during an exposure of $300 \mathrm{~s}$ with Micro-X. Spectral features indicate the presence of cool matter with now resolved Fe K line states and an Fe K edge. P Cygni line features from highly ionized Fe XXV and XXVI ions contain possibly narrow disk emission Doppler lines in addition to the prominent blueshifted line absorption from its powerful disk wind.

addition, one can detect an offset of $>100 \mathrm{~km} / \mathrm{sec}$ between a central cool component and a "background" hot component, which would comprise a novel way of measuring the M87 "peculiar velocity."

The range of temperatures is unambiguously determined and a sophisticated analysis using constraints from higher angular resolution imaging spectroscopy should enable the first substantial progress toward quantifying in detail how the thermal structure departs from that expected for a simple cooling flow. Micro-X will make important and unique contributions to the study of clusters of galaxies and will complement the existing data sets from our current orbiting X-ray observatories.

\subsection{X-ray Binaries}

In spite of much study, understanding of the geometry of the gas flows and circumstellar gas in X-ray binaries remains uncertain. Many of the fundamental issues can only be disentangled via high resolution spectroscopy, ${ }^{31}$ particularly of the Fe K complex, the strongest spectral feature in these objects. It is difficult to perform these observations using the Chandra gratings due to the effects of detector pulse pile-up in the first order and because of the limited energy resolution at $\mathrm{E}>2 \mathrm{keV}$. While several classes of X-ray binaries are bright enough to be observed with Micro-X (e.g. pulsars, low mass x-ray binaries and neutron star transients), we will concentrate on the brightest objects: black hole transients and Cir X-1 (Fgure 7). Some of these sources show evidence for outflow in the form of radio jets, and also show absorption by highly ionized iron. In at least one case, H1743-322, these iron absorption lines are blueshifted by a detectable amount, $700-800 \mathrm{~km} / \mathrm{s}$, providing clear evidence of outflow. Outflows are surprising, since we think these sources are fueled by accretion, and the implied mass outflow rates would exceed the accretion rate if the flows are steady and spherically symmetric. If so, it would provide evidence for electromagnetic extraction of energy from a rotating black hole, a process which has been discussed theoretically but not yet clearly detected. Micro-X simulations of Cir X-1 and GRS 1915, based on the lower resolution Chandra data, demonstrate that the outflow velocity in the Fe XXVI Ly- $\alpha$ line can be 
determined to within $450 \mathrm{~km} / \mathrm{s}$, comparable to the assumed line width. In addition, for Cir X-1 the shape of the $P$ Cygni line profiles can be determined and the presence of narrow disk lines can be inferred.

Study of the bright Fe $\mathrm{K}$ lines from these sources can be accommodated by a future modification of the instrument to incorporate the $3.5 \mathrm{~m}$ focal length ASCA flight spare mirror, which requires only minor modifications to the optical bench.

\section{CONCLUSION}

The scientific potential of the X-ray microcalorimeter for revealing high-precision spectral diagnostics is still largely unfulfilled. Although physically modest compared with Constellation-X or NeXT, the Micro-X payload will go a long way toward providing valuable experience and solutions to the practical problems of deploying TES microcalorimeters in space. By confronting the real issues of producing the payload and flying it, we will achieve a flight TES array, robust and compact electronics unique to these sensors, and obtain astrophysically rich data with a TES microcalorimeter. The science return from this mission, as described in this paper, will be substantial. Micro-X will obtain the first imaging X-ray microcalorimeter spectra from astrophysical objects, give the community a sampling of what future orbiting mission will accomplish, and serve as a pathfinder for Constellation-X.

Work by the University of California LLNL was performed under the auspices of the D.o.E. under contract No. W-7405-Eng-48.

\section{REFERENCES}

1. J. N. Ullom, J. A. Beall, W. B. Doriese, W. D. Duncan, L. Ferreira, G. C. Hilton, K. D. Irwin, C. D. Reintsema, and L. R. Vale, "Optimized transition-edge $\mathrm{x}$-ray microcalorimeter with $2.4 \mathrm{eV}$ energy resolution at 5.9 keV," Applied Physics Letters 87, pp. 4103-+, Nov. 2005.

2. M. A. Lindeman, S. Bandler, R. P. Brekosky, J. A. Chervenak, E. Figueroa-Feliciano, F. M. Finkbeiner, R. L. Kelley, T. Saab, C. K. Stahle, and D. J. Talley, "Performance of compact TES arrays with integrated highfill-fraction X-ray absorbers," Nuclear Instruments and Methods in Physics Research A 520, pp. 411-413, 2004.

3. K. D. Irwin, G. C. Hilton, D. A. Wollman, and J. M. Martinis, "X-ray detection using a superconducting transition-edge sensor microcalorimeter with electrothermal feedback," Applied Physics Letters 69, pp. 19451947, Sept. 1996.

4. J. N. Ullom, W. B. Doriese, G. C. Hilton, J. A. Beall, S. Deiker, W. D. Duncan, L. Ferreira, K. D. Irwin, C. D. Reintsema, and L. R. Vale, "Characterization and reduction of unexplained noise in superconducting transition-edge sensors," Applied Physics Letters 84, pp. 4206-4208, May 2004.

5. P. A. J. de Korte, J. Beyer, S. Deiker, G. C. Hilton, K. D. Irwin, M. Macintosh, S. W. Nam, C. D. Reintsema, L. R. Vale, and M. E. Huber, "Time-division superconducting quantum interference device multiplexer for transition-edge sensors," Review of Scientific Instruments 74, pp. 3807-3815, Aug. 2003.

6. K. D. Irwin, M. D. Audley, J. A. Beall, J. Beyer, S. Deiker, W. Doriese, W. Duncan, G. C. Hilton, W. Holland, C. D. Reintsema, J. N. Ullom, L. R. Vale, and Y. Xu, "In-focal-plane SQUID multiplexer," Nuclear Instruments and Methods in Physics Research A 520, pp. 544-547, Mar. 2004.

7. C. D. Reintsema, J. Beyer, S. W. Nam, S. Deiker, G. C. Hilton, K. Irwin, J. Martinis, J. Ullom, L. R. Vale, and M. Macintosh, "Prototype system for superconducting quantum interference device multiplexing of large-format transition-edge sensor arrays," Review of Scientific Instruments 74, pp. 4500-4508, Oct. 2003.

8. W. B. Doriese, J. A. Beall, W. D. Duncan, L. Ferreira, G. C. Hilton, K. D. Irwin, C. D. Reintsema, J. N. Ullom, L. R. Vale, , and Y. Xu, "Progress toward kilopixel arrays: 3.8 ev microcalorimeter resolution in 8-channel squid multiplexer," NIMPA , in press.

9. D. McCammon, R. Almy, E. Apodaca, W. Bergmann Tiest, W. Cui, S. Deiker, M. Galeazzi, M. Juda, A. Lesser, T. Mihara, J. P. Morgenthaler, W. T. Sanders, J. Zhang, E. Figueroa-Feliciano, R. L. Kelley, S. H. Moseley, R. F. Mushotzky, F. S. Porter, C. K. Stahle, and A. E. Szymkowiak, "A high spectral resolution observation of the soft x-ray di?use background with thermal detectors," ApJ 576, pp. 188-203, 2002. 
10. F. S. Porter, R. Almy, E. Apodaca, E. Figueroa-Feliciano, M. Galeazzi, R. Kelley, D. McCammon, C. K. Stahle, A. E. Szymkowiak, and W. T. Sanders, "Observations of the soft X-ray background with the XQC microcalorimeter sounding rocket," Nuclear Instruments and Methods in Physics Research A 444, pp. 175179, 2000.

11. F. B. S. Paerels and S. M. Kahn, "High-Resolution X-Ray Spectroscopy with CHANDRA and XMMNEWTON," ARAESA 41, pp. 291-342, 2003.

12. M. Güdel, M. Audard, F. Reale, S. L. Skinner, and J. L. Linsky, "Flares from small to large: X-ray spectroscopy of Proxima Centauri with XMM-Newton," A\& A 416, pp. 713-732, Mar. 2004.

13. J. R. Peterson, S. M. Kahn, F. B. S. Paerels, J. S. Kaastra, T. Tamura, J. A. M. Bleeker, C. Ferrigno, and J. G. Jernigan, "High-Resolution X-Ray Spectroscopic Constraints on Cooling-Flow Models for Clusters of Galaxies," ApJ 590, pp. 207-224, June 2003.

14. D. M. Crenshaw, S. B. Kraemer, and I. M. George, "Mass Loss from the Nuclei of Active Galaxies," ARAESA 41, pp. 117-167, 2003.

15. A. Pawl, A. E. Evrard, and R. A. Dupke, "Detecting Intracluster Gas Motion in Galaxy Clusters: Mock Astro-E2 Observations," ApJ 631, pp. 773-784, Oct. 2005.

16. W. Forman, P. Nulsen, S. Heinz, F. Owen, J. Eilek, A. Vikhlinin, M. Markevitch, R. Kraft, E. Churazov, and C. Jones, "Reflections of Active Galactic Nucleus Outbursts in the Gaseous Atmosphere of M87," ApJ 635, pp. 894-906, Dec. 2005.

17. K. A. Flanagan, "Plasma Diagnostics with X-Ray Emission Lines of Puppis A.," MIT Ph.D. Thesis, 1990.

18. C. R. Canizares and P. F. Winkler, "Evidence for elemental enrichment of Puppis A by a Type II supernova," ApJL 246, pp. L33-L36, May 1981.

19. U. Hwang, K. A. Flanagan, and R. Petre, "Chandra X-Ray Observation of a Mature Cloud-Shock Interaction in the Bright Eastern Knot Region of Puppis A," ApJ 635, pp. 355-364, Dec. 2005.

20. R. I. Klein, K. S. Budil, T. S. Perry, and D. R. Bach, "The Interaction of Supernova Remnants with Interstellar Clouds: Experiments on the Nova Laser," ApJ 583, pp. 245-259, Jan. 2003.

21. P. Beiersdorfer, R. E. Olson, G. V. Brown, H. Chen, C. L. Harris, P. A. Neill, L. Schweikhard, S. B. Utter, and K. Widmann, "X-Ray Emission Following Low-Energy Charge Exchange Collisions of Highly Charged Ions," Physical Review Letters 85, pp. 5090-5093, Dec. 2000.

22. M. W. Wise and C. L. Sarazin, "Charge transfer and X-ray emission from supernova remnants," ApJ 345, pp. 384-392, Oct. 1989.

23. A. P. Rasmussen, E. Behar, S. M. Kahn, J. W. den Herder, and K. van der Heyden, "The X-ray spectrum of the supernova remnant 1E 0102.2-7219," A\&\&A 365, pp. L231-L236, Jan. 2001.

24. R. Petre, G. A. Kriss, P. F. Winkler, and C. R. Canizares, "A high-resolution X-ray image of Puppis A Inhomogeneities in the interstellar medium," ApJ 258, pp. 22-30, July 1982.

25. K. Tamura, "ASCA observation of the Puppis A supernova remnant," Ph.D. Thesis, 1994.

26. I. Sakelliou, J. R. Peterson, T. Tamura, F. B. S. Paerels, J. S. Kaastra, E. Belsole, H. Böhringer, G. Branduardi-Raymont, C. Ferrigno, J. W. den Herder, J. Kennea, R. F. Mushotzky, W. T. Vestrand, and D. M. Worrall, "High resolution soft X-ray spectroscopy of M 87 with the reflection grating spectrometers on XMM-Newton," AËA 391, pp. 903-909, Sept. 2002.

27. J. S. Kaastra, T. Tamura, J. R. Peterson, J. A. M. Bleeker, C. Ferrigno, S. M. Kahn, F. B. S. Paerels, R. Piffaretti, G. Branduardi-Raymont, and H. Böhringer, "Spatially resolved X-ray spectroscopy of cooling clusters of galaxies," A\&广A 413, pp. 415-439, Jan. 2004.

28. K. Matsushita, E. Belsole, A. Finoguenov, and H. Böhringer, "XMM-Newton observation of M 87. I. Singlephase temperature structure of intracluster medium," A\& $A$ 386, pp. 77-96, Apr. 2002.

29. K. Matsushita, A. Finoguenov, and H. Böhringer, "XMM observation of M 87. II. Abundance structure of the interstellar and intergalactic medium," A\&SA 401, pp. 443-461, Apr. 2003.

30. S. Molendi, "On the Temperature Structure of M87," ApJ 580, pp. 815-823, Dec. 2002.

31. J. C. Lee, C. S. Reynolds, R. Remillard, N. S. Schulz, E. G. Blackman, and A. C. Fabian, "High-Resolution Chandra HETGS and Rossi X-Ray Timing Explorer Observations of GRS 1915+105: A Hot Disk Atmosphere and Cold Gas Enriched in Iron and Silicon," ApJ 567, pp. 1102-1111, Mar. 2002. 